This is an electronic reprint of the original article. This reprint may differ from the original in pagination and typographic detail.

Author(s): Ekman, Axel; Miettinen, Arttu; Tallinen, Tuomas; Timonen, Jussi

Title: $\quad$ Contact formation in random networks of elongated objects

Year: $\quad 2014$

Version:

Please cite the original version:

Ekman, A., Miettinen, A., Tallinen, T., \& Timonen, J. (2014). Contact formation in random networks of elongated objects. Physical Review Letters, 113(26), Article 268001. https://doi.org/10.1103/PhysRevLett.113.268001

All material supplied via JYX is protected by copyright and other intellectual property rights, and duplication or sale of all or part of any of the repository collections is not permitted, except that material may be duplicated by you for your research use or educational purposes in electronic or print form. You must obtain permission for any other use. Electronic or print copies may not be offered, whether for sale or otherwise to anyone who is not an authorised user. 


\title{
Contact formation in random networks of elongated objects
}

\author{
A. Ekman, ${ }^{*}$ A. Miettinen,$^{\dagger}$ T. Tallinen,$^{\ddagger}$ and J. Timonen ${ }^{\S}$ \\ Department of Physics, University of Jyväskylä
}

(Dated: December 8, 2014)

\begin{abstract}
The effect of steric hindrance is an important aspect of granular packings as it gives rise to, e.g., limitations on the densities of ordered and disordered packings, both of which are essentially defined by the geometry of the constituents. Here we focus on random packing of rods via deposition and their distributions of contact number and segment length. Such statistical properties are relevant for mechanical properties of the structures, but the (quite large) steric effects on them have not been addressed in previous studies. We therefore develop a theory that describes the statistical properties of rod packings, while taking into account that the deposited rods cannot overlap and thus induce steric hindrances. The distributions derived from the theory are compared with experimental results and numerical simulations of networks constructed via deposition. Results explain the non-Poisson statistics observed in the experiments and show that the induced steric range of the rods can be large compared to their diameter and decreases with compactification of the pile, implying local orientational ordering of the structure.
\end{abstract}

There is a wide variety of ubiquitous materials based on random packing of elongated objects, such as, for instance, colloidal suspensions, granular powders, polymer networks, paper, and filter materials. In addition, new similar materials are being developed, such as carbon nanotube films in flexible electronics [1] and fibrous scaffolds in tissue engineering [2], which are essentially formed by random deposition of fibers (very thin rods). Understanding the geometry and structural properties of such random packings, and how they are related to, e.g., their mechanical or electrical properties, is important from the basic scientific point of view, but especially for their engineering applications.

Random packing of stiff constituents is governed predominantly by their geometry. For example, random packing of spheres can maximally reach a solids content of $\phi_{\mathrm{s}} \approx 0.64$ corresponding to the Bernal sphere packing [3] with a mean coordination number of 6 , i.e., the mean number of contacts per particle. For elongated particles packing density $\phi_{\mathrm{s}}$ approaches zero as the aspect ratio of the particles increases [4], and the coordination number approaches about 10 in the limit of large aspect ratios [5]. Even though particle correlations play a significant role in the packing of low-aspect-ratio particles $[6,7]$, averaged quantities of isotropic random packings of high aspect-ratio particles (rods), such as density and mean coordination number, can well be described by the random-contact model [4] which assumes uncorrelated contacts between rods and involves therefore only simple excluded-volume arguments. That model does not, however, describe correctly the distribution of, e.g., contact numbers in individual rods, which has been observed to differ $[5,8]$ from the Poisson distribution, i.e., the result of an uncorrelated process. It thus seems that contacts between rods in their random packings are not uncorrelated after all, and that this effect is rather important.

Random networks are typically formed of fibers by deposition or some other random-packing process. So far analysis of their mechanics and geometry has mainly been based on the properties of relatively simple twodimensional (2D) random networks made of infinitely long lines. Motivation to this starting point has been that many such networks are formed by unidirectional compression under gravity (e.g., by sedimentation of a dilute suspension) or otherwise have a predominantly planar orientation. 2D networks of infinite lines are fairly well understood theoretically $[9,10]$ and have an uncorrelated structure: Poissonian distribution of contact numbers along individual lines and a negative-exponential distribution of segment lengths. Although experimental results have a negative-exponential tail in the segmentlength distribution, significant deviations from it have been found at short segment lengths [11]. Distribution of segment lengths is particularly important for the mechanical properties of three-dimenstional (3D) networks of fibers $[12,13]$.

In order for a $2 \mathrm{D}$ deposited network of fibers to grow vertically, or their 3D isotropic packings to be stable, hard-core interactions between fibers must also be accounted for. These interactions introduce steric hindrances for fibers, and thus contacts in their packings are not necessarily uncorrelated. Although the effect of steric hindrance on global properties such as density has been investigated previously $[14,15]$, statistical properties have remained elusive. In this article we introduce a theoretical framework to account for these hard-core interactions and give distributions for the both coordination number and segment length. Comparison of theoretical results with those of experiments and numerical simulations suggest that both of the above distributions can be understood on this theoretical basis: correlation between contacts is related to the steric hindrance between fibers, and these induce local orientational order in the structure.

To clarify why Poisson statistics is an inevitable consequence if hard-core interactions are not taken into ac- 
count, we start by considering a $2 \mathrm{D}$ network composed randomly of rectangular objects of finite length $l_{\mathrm{f}}$ and non-zero width $w_{\mathrm{f}}$. Given two rectangles of the same size, the probability that they cross is proportional to their excluded area, i.e., the area where the center point of the other rectangle must be placed such that the two rectangles would intersect. For two rectangles with a difference of $\theta$ in their orientation, this area of intersection is given by $A_{\mathrm{i}}=\left(l_{\mathrm{f}} \sin \theta+w_{\mathrm{f}}+w_{\mathrm{f}} \cos \theta\right)\left(l_{\mathrm{f}}+w_{\mathrm{f}} \sin \theta+\right.$ $\left.l_{\mathrm{f}} \cos \theta\right)-\left(l_{\mathrm{f}}^{2}-w_{\mathrm{f}}^{2}\right) \sin \theta \cos \theta[16]$.

Now consider a deposited network of $N_{f}$ such rectangles on area $A$. For an individual rectangle, all the other $N_{f}-1$ rectangles have a probability of $A_{\mathrm{i}} / A$ to intersect with it. Therefore the total number of crossings on this rectangle can be expressed in the form $\langle C\rangle=\left(N_{f}-1\right)\left\langle A_{i}\right\rangle / A$. For a dense network $\left(N_{\mathrm{f}} \gg 1\right)$ of narrow rectangles $\left(l_{\mathrm{f}} \gg w_{\mathrm{f}}\right)$ with uniformly distributed centers and crossing angles, this result is consistent with that of Korte and Callmes [10], and we find that

$$
\langle C\rangle=\frac{2 l_{\mathrm{f}}^{2}}{\pi} \frac{N_{\mathrm{f}}}{A} .
$$

For uniformly distributed centers and orientations of the rectangles, contacts can be considered to be formed by successive Bernoulli trials with a probability of $\left\langle A_{\mathrm{i}}\right\rangle / A$, and thus, if an average $\langle C\rangle>0$ exists for a large system, the coordination number of a rectangle is Poisson distributed. This results approximately (exactly in the case of random infinitely long lines [9]) in an exponential distribution of segment lengths. The same approach of uncorrelated contacts (i.e., soft-core objects), which only involves the excluded volume, can be extended to other dimensions. For example, Onsager [17] has given the excluded volume in 3D for both regular and sphericallycapped cylinders. Also in these cases the random placement of the constituents in space can be considered as successive Bernoulli trials, and the resulting distribution of coordination number would be Poissonian.

Real packings or networks are not, however, formed by uncorrelated packings of soft-core objects. They are formed by, e.g., deposition, and hard-core interactions are needed between fibers so that these structures can grow vertically. We will thus consider packing (networks) of randomly deposited fibers, in which their non-zero diameter will necessarily involve steric hindrances. Even if these fibers would be infinitely long, a newly deposited fiber will not make contact with all previously deposited fibers as in the 2D networks of infinitely long lines (thus Eq. (1) will always overestimate the coordination number). Instead, the newly deposited fiber makes contact with a subset of fibers, which can still be considered random.

We base our model on the assumption that the fiberfiber contacts in a deposited network may be divided so that they are either on the top or bottom side of a fiber. This division is possible if the fibers were deposited one

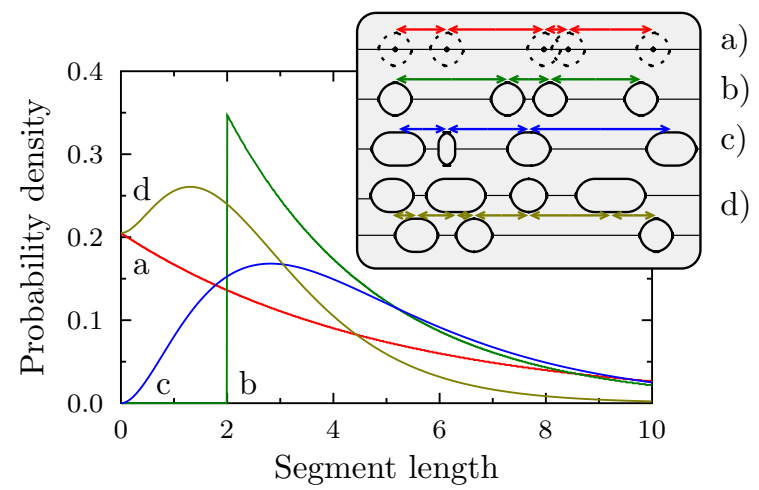

FIG. 1: Schematic illustration of different models for the contact forming process, and the resulting segment length distributions. Poissonian, i.e., soft core (red, a), Tonks gas (green, b), disperse Tonks gas (blue, c), and two superposed disperse Tonks gases (olive, d).

by one, and had a high enough friction to prevent them from sliding below previously deposited fibers. Furthermore, we assume that the contact formation on either side of the fibers is random, but sterically hindered by the hard-core interactions between adjacent fibers. This means that we can treat the two contact-forming processes, viz.: contacts on the either top or bottom sides of the fibers, as two separate hard-core systems. The final contacts are then obtained by superposing these two (one-sided) processes (Fig. 1c) on the same fiber, resulting in a two-sided process (contacts on the both top and bottom sides of the fibers, Fig. 1d).

If the fibers would always cross at right angles, the process of contact formation on one side of a fiber is sterically hindered such that the minimum length between two contact centers would be $w_{\mathrm{f}}$ (contacts would be impenetrable spheres of diameter $w_{\mathrm{f}}$ ). A system that describes this kind of process is the Tonks gas of hard spheres $[18,19]$. By treating the contacts on both sides of the fibers as separate linear gases of hard spheres (contacts) of diameter $d=w_{\mathrm{f}}$ in a one-dimensional (1D) space of length $L=l_{\mathrm{f}}$, we have available both the probabilitydensity function (PDF) of segment lengths [18] (distribution of inter-particle distances in the Tonks gas, Fig. $1 \mathrm{~b})$, and the distribution of coordination numbers [20] for this one-sided process. In reality, the steric hindrances of one-sided contacts are not simple hard-core limits, but they will depend, e.g., on the crossing angles of the fibers in contact. If two fibers in contact on the same side of the fiber differ in their orientation, they can be sterically hindered well beyond the fiber width $w_{\mathrm{f}}$, similar to the system in Ref. [21]. In addition, cylindrical rods are not strictly two-sided, which means that contacts can form anywhere on their perimeters so that rod width is not an exact lower bound for the lengths of the steric hindrance. The main idea is, however, to classify contacts 
into distinct (1D) groups, and for simplicity we consider here distinction into contacts on the top or bottom sides of the fibers.

For the segment-length distribution of one-sided contacts, the original derivation of Tonks [18] can easily be extended to polydisperse gases with particle sizes $d_{i}$ (Fig. 1c, details in SI) and we find that

$$
P\left(l_{\mathrm{s}} \in[x, x+\mathrm{d} x]\right)=\mathrm{d} x \int_{0}^{x} f_{d_{12}}(y) \mu e^{-\mu(x-y)} \mathrm{d} y,
$$

where $f_{d_{12}}$ is the PDF for the combined steric effect of two adjacent fibers, such that $P\left(d_{1} / 2+d_{2} / 2 \in[y, y+\mathrm{d} y]\right)=$ $f_{d_{12}}(y) \mathrm{d} y$, and $\mu$ is process frequency in the reduced space $\mu=\langle C\rangle /(L-\langle C\rangle\langle d\rangle)=\left(\left\langle l_{\mathrm{s}}\right\rangle-\langle d\rangle\right)^{-1}$. Details of the choice of $f_{d_{12}}$ can be found in SI.

The final segment-length distribution is now obtained by superposing the two contact-forming processes (contacts on the both top and bottom sides of the fibers, Fig. 1d). As the distances ('segment lengths') between particle centers in a Tonks gas are uncorrelated this process can be thought of as a renewal process (it is not strictly true, due to correlations between adjacent segment lengths, but has no effect on the resulting distribution as explained in SI), and the cumulative distribution function $(\mathrm{CDF})$ of segment lengths $\left(F_{\mathrm{p}}(x)\right)$ of $p$ superposed processes is given by [22]

$$
1-F_{\mathrm{p}}(x)=\left(1-F_{1}(x)\right)\left[\frac{\langle C\rangle}{L} \int_{x}^{\infty} 1-F_{1}(s) \mathrm{d} s\right]^{p-1},
$$

where $F_{1}(x)$ is the CDF of the one-sided process (contacts on the either top or bottom side of the fibers). Consequently, the PDF of segment lengths for two combined processes is given by $f_{l_{\mathrm{s}}}(x)=\frac{\mathrm{d}}{\mathrm{d} x} F_{2}(x)$.

For the coordination number, no solution was found for the disperse Tonks gas, so we had to approximate the system as a homogeneous Tonks gas for which the coordination-number distribution is given by [20],

$$
P_{1}(C=N)=\frac{\mu L\left[\mu d\left(\frac{L}{d}-N+1\right)\right]^{N}}{\langle C\rangle N !} e^{-\mu d(L / d+1-N)} .
$$

Distribution of the coordination number of all contacts along a fiber can be obtained by superposing the two processes. Exact solutions for $n=\{2,3, \ldots\}$ superposed processes are given by the convolutions

$$
P_{n}(C=N)=P_{1}(C=N) \otimes P_{n-1}(C=N) .
$$

No analytical solution was found for these convolutions directly for any $n>1$, and thus they were solved numerically when necessary.

To test the distribution of the coordination number (Eq. (5)) against experimental data, we used the results of Blouwolff and Fraden [5], who studied packing of essentially rigid rods. The measured coordination-number

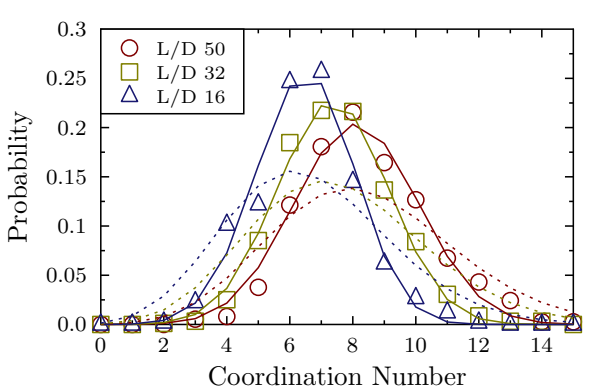

FIG. 2: Distributions of the coordination number of random packings of rods for different aspect ratios, (L/D), together with their best fits by the two-sided Tonks gas distributions (solid lines) and by Poisson distributions (dashed lines). The experimental data are from Blouwolff and Fraden [5].

TABLE I: Average coordination number and particle-size of the fitted two-sided Tonks-gas distributions for non-compactified (nonc.) and compactified (comp.) packings of rods shown in Fig. 2, and experimental results for short-cut spaghetti. All lengths are scaled with rod diameter $D$.

\begin{tabular}{c|cc|cc|cc|c|}
\hline L & \multicolumn{2}{|c|}{50} & \multicolumn{2}{c|}{32} & \multicolumn{2}{c|}{16} & 12.3 \\
& nonc. comp. & nonc. & comp. & nonc. comp. & spag. \\
C & 8.1 & 9.7 & 7.4 & 9.7 & 6.5 & 7.0 & 9.4 \\
d & 4.2 & 2.7 & 3.4 & 2.8 & 2.1 & 1.7 & 0.8 \\
d/L & 0.08 & 0.05 & 0.11 & 0.09 & 0.13 & 0.11 & 0.06 \\
\hline
\end{tabular}

distributions were fitted by the distribution for two-sided contacts (Eq. (5)) using the range of steric interaction $(d)$ and the mean contact number $(\langle C\rangle)$ as the fitting parameters. For comparison, a fit by Poissonian distribution was also made, using the mean coordination number, $\langle C\rangle$, as the fitting parameter. It is evident from Fig. 2 that the experimental and theoretical distributions agree very well, and that they are quite different from a Poissonian distribution, i.e., the uncorrelated-contacts approximation. From the results of these fits (Table I), we can draw two conclusions. First, the hard-core diameter $d$ decreases when the packing of rods is compactified. Second, as the aspect ratio increases, the relative hard-core diameter $d / L$ decreases. It remains unclear, however, if the effect of hard cores vanishes in the limit of diverging aspect ratio. For more comments on these conclusions see below.

To test the segment-length distributions against experimental data, a pile of raw, short-cut spaghetti was imaged with x-ray tomography. Individual rods of spaghetti were segmented from each other, which allowed determination of the coordination numbers and segment lengths in each rod, and thus their distributions. These measured 


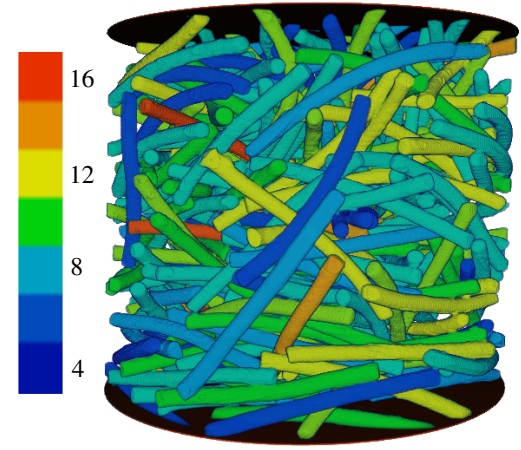

(a)

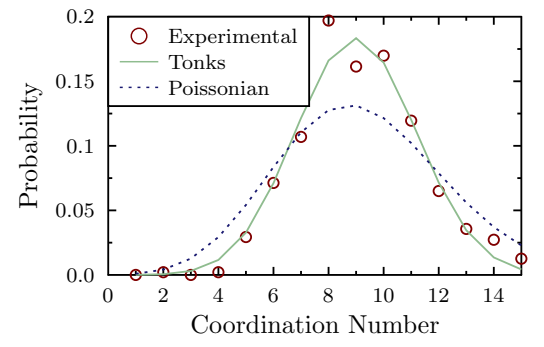

(b)

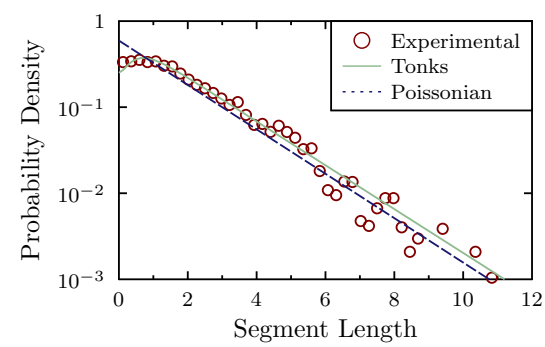

(c)

FIG. 3: (a) A random pile of raw (short-cut) spaghetti as imaged by x-ray tomography. Color indicates the coordination number of the individual rods of spaghetti. (b) Distribution of coordination numbers for this pile. (c) Distribution of segment lengths for this pile (scaled by rod width). So as to minimize the effect of finite length, contacts at the ends of each rod of spaghetti were not included.

distributions were then fitted by the corresponding theoretical results, both for the coordination number (Eq. (5)) and the segment lengths (Eq. (S20)), shown in Figs. $3 \mathrm{~b}$ and $3 \mathrm{c}$, respectively. Here the fitting parameters were the range of steric interaction $(\langle d\rangle)$ and the mean contact number $(C$, for $P(C))$, or the process frequency ( $\mu$, for $\left.P\left(l_{\mathrm{s}}\right)\right)$. The measured distributions deviate clearly from Poissonian and negative-exponential distributions.

For the pile of short-cut spaghetti, division of contacts into two subgroups, viz.: 'top' and 'bottom' contacts, was not well defined, and thus only the 'two(or more)-sided' segment-length distributions could be analyzed (i.e., all contacts were included in the analysis). Because of this, distributions of segment lengths were also determined for numerically deposited 3D packings (networks) of thin rods (fibers) [23]. In the simulated networks, fibers were deposited one by one so that contacts in each fiber could be classified into top and bottom contacts by the chronological order of the fibers (see SI, especially Fig. S7, for details and visualization). We could thus easily determine the one-sided (cf. Eq. (S13)) as well as the twosided (cf. Eq. (S20)) distributions of segment lengths. Both these distributions were fitted by theoretical ones (Fig. 4) using the range of steric interaction $(\langle d\rangle)$ and the process frequency $(\mu)$ as the fitting parameters. The two-sided division of contacts was in very good agreement with the numerical result, and the effect of steric hindrances is evident as the distributions of segment lengths deviate notably from negative-exponential distributions.

It is evident from the results shown in Table I that the steric hindrance $(d)$ is larger than the rod diameter for rods of high aspect ratios. This implies that, although the non-zero diameter of the rods induces hardcore restrictions, the actual steric hindrance has a fiberorientation-dependent component. Decrease of this hin-

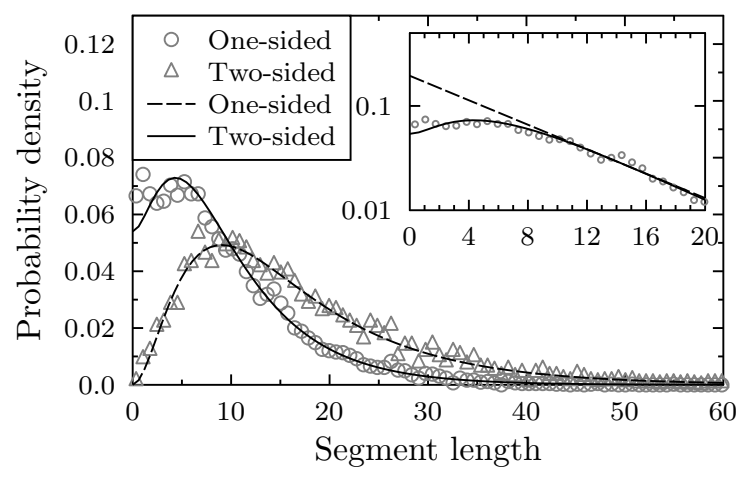

FIG. 4: Distributions of segment lengths between contacts on one side of a fiber and on both sides of fibers in a deposited network. The solid and dashed

lines show the best fits by Eqs. (S13) and (S20), respectively. The inset shows a comparison between the two-sided distribution (all contacts) and a negative-exponential distribution (dashed line). Although the distribution has the characteristic negative-exponential tail, there is a notable deviation from that distribution at small segment lengths.

drance upon compactification of the packing is suggestive of some kind of orientational ordering of adjacent crossing rods, i.e., neighboring rods become more parallel on the average. So as to test the existence of local orientational order, we determined the distribution of difference between the crossing angles in two adjacent contacts along individual fibers. The results (see SI for details) indicate that a local nematic order exists between adjacent contacts along a rod even though the global orientation of the packings is isotropic.

We introduced a theoretical approach for the descrip- 
tion of random packing of rods (or, more generally, of elongated objects), which accounts for the effect of their steric (hard-core) interactions. We considered in particular the distributions of contacts and segment lengths in these packings. It is evident that steric hindrances introduce correlations between adjacent contacts, and the properties of these packings can no longer be described accurately with Poissonian statistics. For the contact number, steric hindrances decrease the variation of its PDF, as the relative volume of the allowed configuration space becomes smaller with increasing steric effects. As for the segment length, steric effects tend to eliminate short segments such that its PDF has a peak near the average steric length, $\langle d\rangle$, which depends on the aspect ratio and flexibility of the fibers. Furthermore, steric effects of (crossing) rods which belong to adjacent contacts along a rod induce local nematic order in the globally isotropic packing. Previous and present experimental results support these findings. They are expected to have implications on the stability and mechanical properties of random packings and networks.

We thank Seth Fraden and Joshua Blouwolff for providing us with their experimental data, all of which could not be deduced from [5], and Michael Thorpe, Robin Stinchcombe, and Lasse Leskelä for useful discussions.

* axel.ekman@jyu.fi

† arttu.i.miettinen@jyu.fi

‡ tuomas.tallinen@phys.jyu.fi

$\S$ jussi.t.timonen@jyu.fi

[1] Q. Cao and J. A. Rogers, Advanced Materials 21, 29 (2009).

[2] C. P. Barnes, S. A. Sell, E. D. Boland, D. G. Simpson, and G. L. Bowlin, Advanced Drug Delivery Reviews 59, 1413 (2007).

[3] J. Bernal, in Proceedings of the Royal Society of London, Series A, Mathematical and Physical Sciences (1964), vol. 280, pp. 299-322.

[4] A. P. Philipse, Langmuir 12, 1127 (1996).

[5] J. Blouwolff and S. Fraden, EPL (Europhysics Let- ters) 76, 1095 (2006), URL http://stacks.iop.org/ $0295-5075 / 76 / i=6 / a=1095$.

[6] C. Song, P. Wang, and H. A. Makse, Nature 453, 629 (2008), ISSN 1476-4687, URL http://dx.doi.org/10. 1038/nature06981.

[7] Y. Jin, J. G. Puckett, and H. A. Makse, Phys. Rev. E 89, 052207 (2014), URL http://link.aps.org/doi/10. 1103/PhysRevE.89.052207.

[8] A. Wouterse, S. Luding, and A. Philipse, Granular Matter 11, 169 (2009), ISSN 1434-5021, 10.1007/s10035009-0126-6, URL http://dx.doi.org/10.1007/ s10035-009-0126-6.

[9] R. E. Miles, Proceedings of the National Academy of Sciences of the United States of America 52, 901 (1964), URL http://www.ncbi.nlm.nih.gov/pmc/ articles/PMC300370/.

[10] O. Kallmes and O. Corte, TAPPI 43, 737 (1960).

[11] J. He, W. J. Batchelor, and R. E. Johnston, Appita Journal 57, 292 (2004).

[12] X.-F. Wu and Y. A. Dzenis, Journal of Applied Physics 98 (2005).

[13] J. A. Åström, J. P. Mäkinen, M. J. Alava, and J. Timonen, Phys. Rev. E 61, 5550 (2000).

[14] N. Pan, Textile Research Journal 63, 336 (1993).

[15] T. Komori and M. Itoh, Textile Research Journal 64, 519 (1994).

[16] I. Balberg, C. H. Anderson, S. Alexander, and N. Wagner, Phys. Rev. B 30, 3933 (1984), URL http://link. aps.org/doi/10.1103/PhysRevB.30.3933.

[17] L. Onsager, Annals of the New York Academy of Sciences 51, 627 (1949).

[18] L. Tonks, Phys. Rev. 50, 955 (1936), URL http://link. aps.org/doi/10.1103/PhysRev.50.955.

[19] A. Münster, Statistical thermodynamics (Springer, 1969).

[20] A. Robledo and J. Rowlinson, Molecular Physics 58, 711 (1986).

[21] Y. Kantor and M. Kardar, Phys. Rev. E 79, 041109 (2009), URL http://link.aps.org/doi/10. 1103/PhysRevE.79.041109.

[22] A. J. Lawrence, Journal of the Royal Statistical Society. Series B (Methodological) 35, pp. 306 (1973), ISSN 00359246, URL http://www.jstor.org/stable/ 2984914.

[23] A. Ekman, A. Miettinen, T. Turpeinen, K. Backfolk, and J. Timonen, Nordic Pulp and Paper Research Journal 27, 270 (2012), URL http://dx.doi.org/10.3183/ NPPRJ-2012-27-02-p270-276. 